\title{
Low-Complexity Robust Beamforming for a Moving Source
}

\author{
Moaaz Mahdi $\quad$ Tarig Ballal ${ }^{\star \star} \quad$ Mohammad Moinuddin ${ }^{\star} \quad$ Tareq Y. Al-Naffouri ${ }^{\star \star} \quad$ Ubaid Al-Saggaf $^{\star}$ \\ * Department of Electrical and Computer Engineering, King Abdulaziz University, Jeddah, KSA \\ ** CEMSE, King Abdullah University of Science and Technology (KAUST), Thuwal, KSA
}

\begin{abstract}
This paper addresses the problem of robust beamforming for a moving source. To cope with the source movement, the regularization (or diagonal loading) parameter needs to be adjusted regularly. The need for repeatedly finding an adequate value of the regularization parameter increases the overall computational complexity. This paper proposes a simple and efficient initialization of Newton's method to find the regularization parameter. The regularization parameter is chosen to minimize the mean-squared-error (MSE) of a regularized least-squares (RLS) problem by solving a certain function. We exploit some properties of this function to develop the method. Simulation results demonstrate that the proposed initialization method can reduce the runtime by $75 \%$.
\end{abstract}

Index Terms-Robust adaptive beamforming, MVDR, generalized sidelobe canceller, diagonal loading.

\section{INTRODUCTION}

Array processing has played a key role in many modern applications such as radar, sonar, radio astronomy, communications and seismology. For example, antenna arrays are essential components in radar systems [1], while hydrophone arrays are widely used in sonar systems [2].

Beamforming, also known as spatial filtering, is a technique used in array processing to receive a signal radiating from a specific direction and suppress signals emerging from other directions [3]. It is well known in the literature that beamforming performance degrades severely in the presence of steering vector errors. This is attributed to improper modeling, miscalibration, pointing error and source motion [4]. Hence, robust and adaptive techniques have been proposed to enhance beamforming performance. These methods include diagonal loading [5], [6], [7] and [8], multiple linear constraints [9], eigenspace projection [10], and the robust Capon beamformers (RCBs) that use ellipsoidal uncertainty sets of the steering vector [11], [12].

In addressing the problem of beamforming for moving sources, most of the work that has been done so far considered acoustic applications while few papers have covered beamforming for moving RF sources. Acoustic signals are wideband with no characteristic wavelength and time delays must be obtained by waveform interpolation. On the other hand, most of the RF signals of interest are narrowband signals with a well-defined nominal wavelength, and time delay can be compensated by a phase shift [13].

A recent acoustic beamforming method is presented in [14]. It is a wavelet-based beamforming method for rotating sources. Acoustic images are produced in the time-frequency domain as a result of direct incorporation of wavelet transform and the Doppler effect into Green's function. Beamforming is achieved as a simple inversion of time-frequency domain.

For RF applications, a Bayesian beamforming approach for a moving target is presented in [4]. The optimal beamformers' coefficient for this technique is the sum of minimum variance distortionless response (MVDR) beamformer at the estimated direction of arrivals (DOAs) weighted by a posterior probability density function (PDF) of these DOAs. A particle filter is used to find this a posteriori PDF.

A sliding window modified loaded sample matrix inversion (LSMI) beamforming algorithm was proposed in [15] for high speed mobile sources. The algorithm is based on recursive vector updating rather than matrix updating. For a sliding window of size $K$ and antenna array of size $N$, The algorithm requires updating $K$ vectors of size $N$, instead of $K$ matrices of size $N \times N$. However, this method uses fixed diagonal loading method, which is not effective for a moving source beamforming.

The proposed beamfoming method in [16] is based on Fractional Fourier transform (FrFT). The beamformer involves optimum FrFT order selection based on the strongest amplitude peak search. However, this search based method is not practical and computationally inefficient [17].

Other methods aim at reducing computational complexity in beamforming. For example, reduced-rank methods are used to provide fast convergence and reduce computational complexity of adaptive beamformers. These methods are based on projecting data onto a low rank subspace. The work in [12] proposes a Krylov-subspace based reduced-dimension robust Capon beamformers. Sensitivity to the signal of interest error (SOI) and array steering vector (ASV) errors is the main disadvantage of the Krylov-subspace methods [12].

In this paper, we aim to develop a robust beamforming where the steering vector is not known precisely due to source movement and DOA estimation errors [18], [19] and [20]. Our beamformer is based on loaded MVDR with data 
collection achieved by sliding the observation window to allow a new snapshot to enter and an old one to leave. This sliding-window process results in a slight change in the regularization (diagonal loading) parameter. Unlike [15] that utilizes a fixed regularization parameter, this work exploits this sliding window setting to persistently update the regularization parameter, as will be explained later.

The remaining of this paper is organized as follows: Section II covers some background material, Section III presents the proposed technique, Section IV presents simulation results while Section V states the conclusion of the paper.

\section{BACKGROUND}

\section{A. Signal model and MVDR beamformer}

Consider a moving source with angular motion described by the following equation:

$$
\theta_{t}=\theta_{\mathrm{o}}+g(t)
$$

where $\theta_{t}(\mathrm{rad})$ is the position of the source at time $t, \theta_{\mathrm{o}}(\mathrm{rad})$ is its initial position and $g(t)$ is a continuous function of time. For linear motion model $g(t)=\omega t$, where $\omega(\mathrm{rad} / \mathrm{sec})$ is the angular velocity. Also consider a uniform linear array (ULA) of $N$ elements receiving a signal from this moving source and $Q$ signals from static interference sources. The $N \times 1$ complex array observation vector can be modeled as [21]

$$
\mathbf{y}_{t}=\beta_{t} \mathbf{a}\left(\theta_{t}\right) s_{t}+\mathbf{v}_{t},
$$

where $\mathbf{a}\left(\theta_{t}\right)$ is the steering vector of the desired source $s_{t}$, the term $\mathbf{v}_{t}=\sum_{i=1}^{Q} \mathbf{a}_{i}[t] s_{i}[t]+\mathbf{n}[t] \in \mathbb{C}^{N}$ is the sum of interference signals, $\mathbf{s}_{i}[t]$, multiplied by their corresponding steering vectors, $\mathbf{a}_{i}[t]$, and additive white Gaussian noise, $\mathbf{n}[t]$. We model the variability in signal amplitude due to distance change using the scalar $\beta_{t}$. Throughout this paper, without loss of generality, we use the following exponential model for amplitude change:

$$
\beta_{t}=e^{-\alpha t}
$$

where $\alpha \in \mathbb{R}, \alpha \geq 0$

The beamformer output at time $t$ is given by [19]

$$
x_{t}=\mathbf{w}_{t}^{H} \mathbf{y}_{t},
$$

where $\mathbf{w}_{t} \in \mathbb{C}^{N}$ is the beamformer weighting coefficients at time $t$. For an MVDR beamformer, the weighting vector is found by solving the following optimization problem [19]:

$$
\mathbf{w}_{\text {opt }}[t]=\underset{\mathbf{w}_{t}}{\operatorname{argmin}} \mathbf{w}_{t}^{H} \mathbf{R}_{t} \mathbf{w}_{t}^{H} \quad \text { s.t. } \mathbf{a}_{t}^{H} \mathbf{w}_{t}=1,
$$

where $\mathbf{R}_{t}=\mathbb{E}\left[\mathbf{y}_{t} \mathbf{y}_{t}^{H}\right]$ is the covariance matrix, and $\mathbf{a}_{t}=$ $\mathbf{a}\left(\theta_{t}\right) \in \mathbb{C}^{N}$ is the steering vector of the desired signal. The solution of (5) is given by [19]

$$
\mathbf{w}_{\text {opt }}[t]=\frac{\mathbf{R}_{t}^{-1} \mathbf{a}_{t}}{\mathbf{a}_{t}^{H} \mathbf{R}_{t}^{-1} \mathbf{a}_{t}}
$$

In practical applications, $\mathbf{R}_{t}$ is unavailable; hence, we replace it with an estimate $\widehat{\mathbf{R}}_{t}$ that is given by

$$
\widehat{\mathbf{R}}_{t}=\frac{1}{K} \sum_{t=1}^{K} \mathbf{y}_{t} \mathbf{y}_{t}^{H}
$$

where $K$ is the number of snapshots.

\section{B. Generalized Sidelobe Canceller (GSC)}

An alternative formulation of (5) is called the generalized sidelobe canceller (GSC) and can be obtained by decomposing the weights $\mathbf{w}$ as follows [7]:

$$
\mathbf{w}_{t}=\mathbf{w}_{q}[t]-\mathbf{B}_{t} \mathbf{w}_{a}[t],
$$

where $\mathbf{w}_{q}[t]=\mathbf{a}_{t} / N$, is a quiescent weight vector and $\mathbf{B}_{t} \in$ $\mathbb{C}^{N \times(N-1)}$ is a blocking matrix that is orthogonal to a, and is chosen such that $\mathbf{B}_{t}^{H} \mathbf{B}_{t}=\mathbf{I}$. By substituting the decomposed $\mathbf{w}_{t}$ (8) in (5) and replacing $\mathbf{R}_{t}$ with $\widehat{\mathbf{R}}_{t}$. The problem can be reformulated as the following unconstrained least squares optimization [7]:

$$
\min _{\mathbf{w}_{a}[t]}\left(\mathbf{B w}_{a}[t]-\mathbf{w}_{q}[t]\right)^{H} \widehat{\mathbf{R}}_{t}\left(\mathbf{B}_{t} \mathbf{w}_{a}[t]-\mathbf{w}_{q}[t]\right)
$$

or

$$
\min _{\mathbf{w}_{a}[t]}\left\|\mathbf{A}_{t} \mathbf{w}_{a}[t]-\mathbf{b}_{t}\right\|^{2}
$$

where $\mathbf{A}_{t} \triangleq \widehat{\mathbf{R}}_{t}^{\frac{1}{2}} \mathbf{B}_{t} \in \mathbb{C}^{N \times N-1}$ and $\mathbf{b}_{t} \triangleq \widehat{\mathbf{R}}_{t}^{\frac{1}{2}} \mathbf{w}_{q}[t]$. The minimization (10) corresponds to the following linear regression model:

$$
\mathbf{b}_{t}=\mathbf{A}_{t} \mathbf{w}_{a}[t]+\mathbf{z}_{t}
$$

where $\mathbf{z} \in \mathbb{C}^{N}$ is an error vector. Since $\widehat{\mathbf{R}}_{t}^{\frac{1}{2}}$ is normally illconditioned, and $\mathbf{b}_{t}$ is noisy, the application of regularization to estimate $\mathbf{w}_{a}[t]$ is preferred. The regularized least squares (RLS) problem is stated as follows:

$$
\min _{\mathbf{w}_{a}[t]}\left\|\mathbf{A}_{t} \mathbf{w}_{a}[t]-\mathbf{b}_{t}\right\|^{2}+\gamma_{t}\left\|\mathbf{w}_{a}[t]\right\|^{2}
$$

After choosing a proper value for $\gamma_{t}$, we can use it in the loaded version of (6) that is given by [7]

$$
\mathbf{w}_{\text {opt }}[t]=\frac{\left(\mathbf{R}_{t}+\gamma_{t} \mathbf{I}\right)^{-1} \mathbf{a}_{t}}{\mathbf{a}_{t}^{H}\left(\mathbf{R}+\gamma_{t} \mathbf{I}\right)^{-1} \mathbf{a}_{t}}
$$

\section{THE PROPOSED METHOD}

In this section, we eliminate the subscript $t$ for simplicity of notations. To obtain the regularization parameter, $\gamma$, we solve the following equation [5]:

$$
\begin{aligned}
f(\gamma) & =\operatorname{tr}\left[\left(\boldsymbol{\Sigma}^{2}+\gamma \mathbf{I}\right)^{-1}\right] \operatorname{tr}\left[\left(\boldsymbol{\Sigma}^{2}+\gamma \mathbf{I}\right)^{-1} \mathbf{U}^{H} \mathbf{b} \mathbf{b}^{H} \mathbf{U}\right] \\
& -N\left[\left(\boldsymbol{\Sigma}^{2}+\gamma \mathbf{I}\right)^{-2} \mathbf{U}^{H} \mathbf{b b}^{H} \mathbf{U}\right]=0,
\end{aligned}
$$

where $\operatorname{tr}($.$) denotes the matrix trace, \mathbf{I}$ is the identity matrix, $\mathbf{U} \in \mathbb{C}^{N \times N}$ and $\boldsymbol{\Sigma}=\operatorname{diag}\left[\sigma_{1}, \sigma_{2}, \cdots \sigma_{N}\right]^{T}$, with $\sigma_{1}>\sigma_{2}>$ $\cdots>\sigma_{N}$ are obtained from the following singular value decomposition (SVD) of $\mathbf{A}$

$$
\mathbf{A}=\mathbf{U} \boldsymbol{\Sigma} \mathbf{V}^{H}
$$

where $\mathbf{V} \in \mathbb{C}^{N-1 \times N}$.

The regularization parameter values that solve Equation (14) minimize the minimum-squared-error (MSE) of the RLS as shown in details in [5]. 
The introduction of the regularization term in (12) aims at providing stability against the ill-conditioning of the matrix A. To reap the full benefit of the regularization process, the regularization parameter $\gamma$ has to be adjusted carefully. The process of setting the regularization parameter can be performed independently at each time point. Several methods exist to achieve this goal (e.g., [22] [7] ). Applying these methods repetitively at each time point may result in unnecessarily increasing the computational complexity of the system. In the following, we present a method to effectively adjust the regularization parameter needed in (12) with significantly reduced computational complexity.

Newton's method, also known as the Newton-Raphson method, is a well-known technique used to find a root of a function [23]. Starting from an initial guess $\gamma^{i=0}$ for the root of (14), the following iterations are carried out:

$$
\gamma^{i+1}=\gamma^{i}-\frac{f\left(\gamma^{i}\right)}{f^{\prime}\left(\gamma^{i}\right)},
$$

where $f^{\prime}\left(\gamma^{i}\right)$ is the derivative of the function. The iterations stop when $\left|f\left(\gamma^{i+1}\right)\right|<\epsilon$.

Our technique relies on some properties of Equation (14) presented in [5]. Specifically, we focus on a situation that guarantees the existence of a unique solution for Equation (14). It is shown that a unique root exists in the interval $\left(-\sigma_{n}, \infty\right)$ if the following sufficient condition is met [5]:

$$
N \operatorname{tr}\left(\boldsymbol{\Sigma}^{2} \mathbf{U}^{H} \mathbf{b b}^{H} \mathbf{U}\right)>\operatorname{tr}\left(\boldsymbol{\Sigma}^{2}\right) \operatorname{tr}\left(\mathbf{U}^{H} \mathbf{b b}^{H} \mathbf{U}\right) .
$$

If $f(\gamma)$ has a positive root, $\gamma^{+}$, and the condition (17) is satisfied, the following two results are valid [5]:

1) $f(\gamma)$ is always negative in the interval $\left[0, \gamma^{+}\right)$, i.e., $f(\gamma) \leq 0$ for $\left[0, \gamma^{+}\right]$

2) $f(\gamma)$ is an increasing function in the interval $\left[0, \gamma^{+}\right]$, i.e., $f^{\prime}(\gamma) \geq 0$ for $\left[0, \gamma^{+}\right]$

Thus, using an initial value $\gamma^{0}$ in the interval $\left(0, \gamma^{+}\right]$, (16) can produce a progressively increasing estimate of $\gamma$. Convergence of (16) occurs when $\gamma^{i+1} \rightarrow \gamma^{+}$; thus, $f\left(\gamma^{i}\right) \rightarrow 0$ and $\gamma^{i+1} \rightarrow$ $\gamma^{i}$. Fig. 1 plots different examples of (14) when condition (17) is satisfied.

In our moving source beamforming scenario, data is collected by a sliding window that allows only the newest snapshot to enter and the oldest one to leave the window. Hence, we expect a slight change in $\gamma^{+}$for each new sliding window. However, starting from $\gamma^{0}=\delta$, where $\delta$ is a small positive value, for each new sliding window would increase the number of iterations that are required for $f(\gamma)$ to converge to a positive root.

We assume that the root of the current sliding window is $\gamma_{\mathrm{o}}^{+}$. The root of the next sliding window is either $\gamma_{\mathrm{r}}^{+}$, where $\gamma_{\mathrm{r}}^{+}>\gamma_{\mathrm{o}}^{+}$, or $\gamma_{1}^{+}$, where $\gamma_{1}^{+}<\gamma_{\mathrm{o}}^{+}$. Fig. 1 (b) plots Equation (14) with these possibilities. We assume the black curve, $f_{\mathrm{o}}(\gamma)$, is (14) calculated from the the data covariance matrix which is estimated from the current sliding window. Using Newton's method, we find the root of $f_{\mathrm{o}}(\gamma)$ with an initial guess $\left(\gamma^{i=0}=\delta\right)$ to obtain the root, $\gamma_{0}^{+}$. If the next sliding window plots the blue curve, $f_{\mathrm{r}}(\gamma)$, we do not need to initialize with $\gamma^{i=0}=\delta$ when using Newton's method. Instead, we examine the derivative, $f_{\mathrm{r}}^{\prime}(\gamma)$ at the previous root, $\gamma_{\mathrm{o}}^{+}$. Since $f_{\mathrm{r}}^{\prime}\left(\gamma_{\mathrm{o}}^{+}\right)>0$ this implies that $\gamma_{\mathrm{o}}$ is a suitable initialization point for $f_{\mathrm{r}}^{\prime}(\gamma)$ to find its root because $\gamma_{\mathrm{o}}^{+}$lies in the interval $\left(0, \gamma_{\mathrm{r}}^{+}\right]$.

If the next sliding window moves to the left from $f_{\mathrm{o}}(\gamma)$, it produces the red curve $f_{1}(\gamma)$. In this case, testing $f_{1}^{\prime}(\gamma)$ at $\gamma_{o}^{+}$reveals a negative sign which implies that $\gamma_{o}^{+}$is not in the interval $\left(0, \gamma_{1}^{+}\right]$. For this scenario, we bisect the interval $\left(0, \gamma_{o}^{+}\right)$and test the sign at $\gamma_{o}^{+} / 2$. If the result is a positive sign, we can use $\gamma_{o}^{+} / 2$ as an initialization value for finding the root of $f_{1}(\gamma)$. Otherwise, we repeat bisecting and testing process until we obtain a positive sign. Algorithm 1 summarizes the proposed initialization.

To further reduce the complexity, we notice that the SVD of A is needed to find a root $\gamma^{+}$in (14). However, repeating the SVD increases the complexity of the system. We propose a different approach to carry out the calculations efficiently. As explained earlier, we calculate $\mathbf{A}$ from the blocking matrix, $\mathbf{B}$, and the estimated covariance matrix, $\widehat{\mathbf{R}}$. Since $\widehat{\mathbf{R}}$ is a positive semidefinite matrix, we can use the eigenvalue decomposition (EVD) as follows:

$$
\widehat{\mathbf{R}}=\mathbf{L S L}^{H}
$$

where $\mathbf{L} \in \mathbb{C}^{N \times N}$ and $\mathbf{S}=\operatorname{diag}\left[s_{1}, s_{2}, \cdots s_{N}\right]^{T}$, with $s_{1}>s_{2}>\cdots>s_{N}$. We choose an arbitrary matrix, $\mathbf{M} \in \mathbb{C}^{(N-1) \times N}$ such that $\mathbf{B}=\mathbf{L} \mathbf{M}^{H}$, or

$$
\mathbf{M}^{H}=\mathbf{L}^{H} \mathbf{B}
$$

Now we can write $\mathbf{A}$ differently as follows:

$$
\begin{aligned}
\mathbf{A} & =\widehat{\mathbf{R}}^{\frac{1}{2}} \mathbf{B} \\
& =\mathbf{L} \mathbf{S}^{\frac{1}{2}} \mathbf{L}^{H} \mathbf{L} \mathbf{M}^{H} \\
& =\mathbf{L} \mathbf{S}^{\frac{1}{2}} \mathbf{M}^{H}
\end{aligned}
$$

Comparing with (15), we notice $\mathbf{L}=\mathbf{U}, \mathbf{S}^{\frac{1}{2}}=\mathbf{\Sigma}, \mathbf{M}=\mathbf{V}$. For each sliding window, we modify $\widehat{\mathbf{R}}$ by adding a rank1 matirx and subtracting a rank-1 matrix. This allows us to use a recursive algorithm, described in [24], to compute the eigenvalues and eigenvectors of $\widehat{\mathbf{R}}$ and use them directly in (14). This reduces the complexity by an order of magnitude from $O\left(N^{3}\right)$ to $O\left(N^{2}\right)$. 


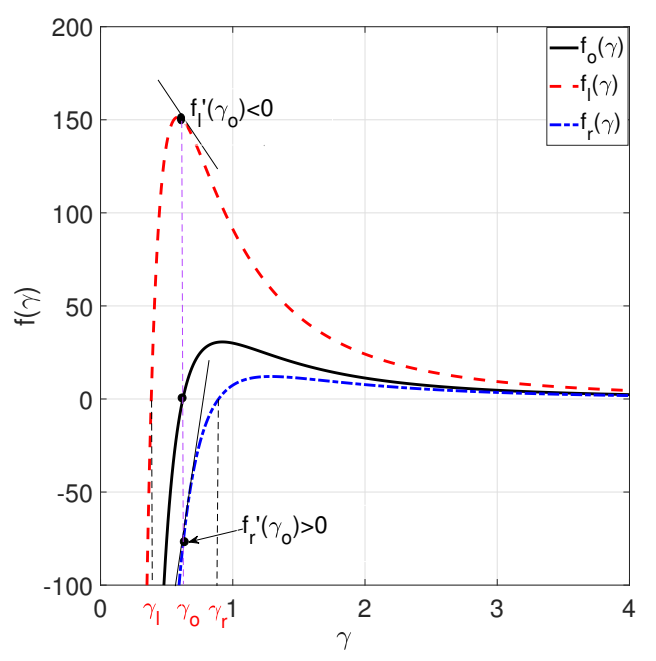

Fig. 1. BPR function (Equation (14)).

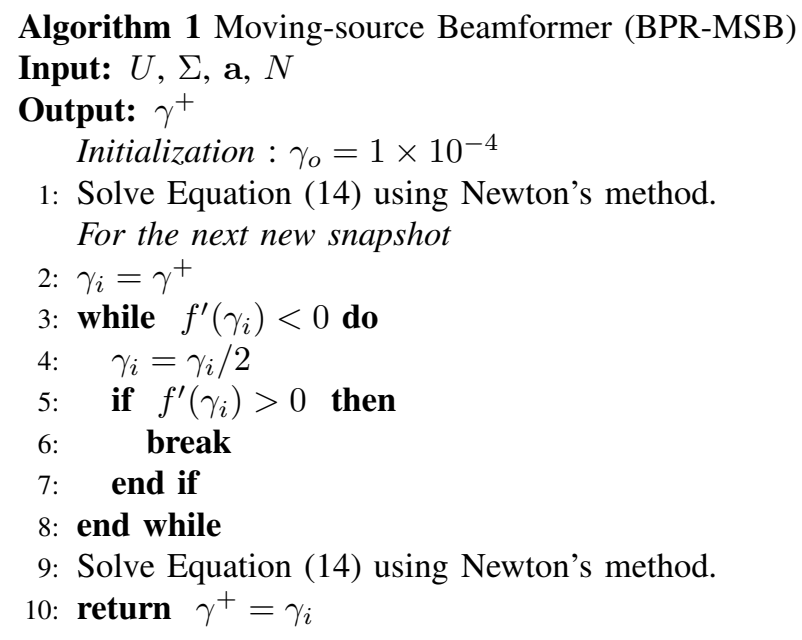

\section{Simulation Results}

Signal-to-interference and noise ratio (SINR) is considered for performance evaluation, which is calculated as follows [19]:

$$
\operatorname{SINR}_{t}=\frac{\sigma_{s}^{2}[t]\left|\mathbf{w}_{t}^{H} \mathbf{a}_{\mathrm{o}}[t]\right|^{2}}{\mathbf{w}_{t}^{H} \mathbf{R}_{i+n}[t] \mathbf{w}_{t}},
$$

where $\sigma_{s}[t]$ is the moving source signal power at time $t, \mathbf{a}_{\mathrm{o}}[t]$ is the actual steering vector of the desired signal at time $t$, and $\mathbf{R}_{i+n}[t]$ is the interference-plus-noise covariance matrix at time $t$.

We assume a ULA of $N=10$ elements that receives an RF signal transmitted by a moving source that starts at $0^{\circ}$ and stops at $60^{\circ}$. During the movement of the source, the signal's amplitude suffers up to $20 \%$ attenuation. There are four interference signals $(Q=4)$ located at fixed positions. $\left[-30^{\circ},-60^{\circ}, 100^{\circ}, 120^{\circ}\right]$ with an interference-to-noiseratio (INR) of $10 \mathrm{~dB}$. The snapshots are collected in a sliding window of size $K=10$. We assume that the DOA of the source that coincides with each snapshot is known with a

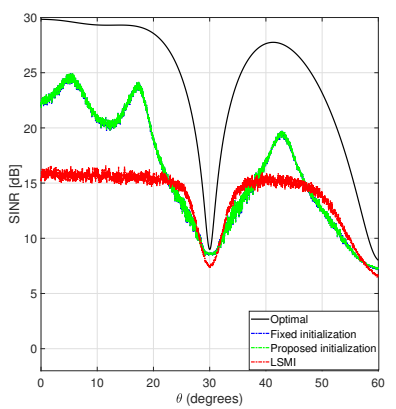

Fig. 2. Beamoforming performance $\left(\mathrm{SNR}=10 \mathrm{~dB}\right.$ at $\left.\theta=0^{\circ}\right)$.

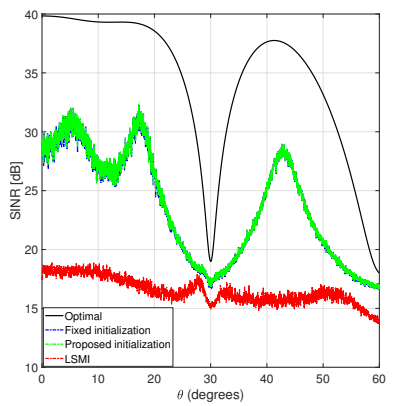

Fig. 3. Beamoforming performance $\left(\mathrm{SNR}=20 \mathrm{~dB}\right.$ at $\left.\theta=0^{\circ}\right)$.

uniformly distributed uncertainty in the interval $\left[-1^{\circ}, 1^{\circ}\right]$. We consider two cases with a signal-to-noise ratio (SNR) equal to $10 \mathrm{~dB}$ and $20 \mathrm{~dB}$ at $\theta=0^{\circ}$.

Fig. 2 shows a performance comparison at $\mathrm{SNR}=10 \mathrm{~dB}$ (at $\theta=0^{\circ}$ ) for the proposed method, the fixed initialization method [5] and the loaded sample matrix inversion (LSMI) method [15]. Both of the proposed and fixed initialization methods dynamically update their diagonal loading during the movement. LSMI method uses a fixed diagonal loading $\gamma_{\mathrm{FL}}=10 \mathrm{~dB}$ [15]. It can be seen that both of the proposed and the fixed initialization methods noticeably outperform LSMI in the range $\left(0^{\circ}, 20^{\circ}\right)$ and around $45^{\circ}$.

Fig. 3 shows the same comparison when $\mathrm{SNR}=20 \mathrm{~dB}$ at $\theta=0^{\circ}$. It can be seen that both of the proposed and the fixed methods, with continuously updated regularization parameters, outperform The LSMI method over the entire.

The results show the benefit of continuously updating the diagonal loading parameter. Both of Fig. 2 and Fig. 3 show the proposed method performance is matching the fixed initialization method performance because they solve (14) for the same $\gamma$. However, the proposed initialization method can save around $75 \%$ of the runtime.

Fig. 4 (a) illustrates complexity comparison in terms of the number of iterations between the fixed initialization and the proposed initialization methods over 50 sliding windows. It is obvious that using the proposed technique saves a lot of iterations required to converge to the desired value of the regularization parameter. 


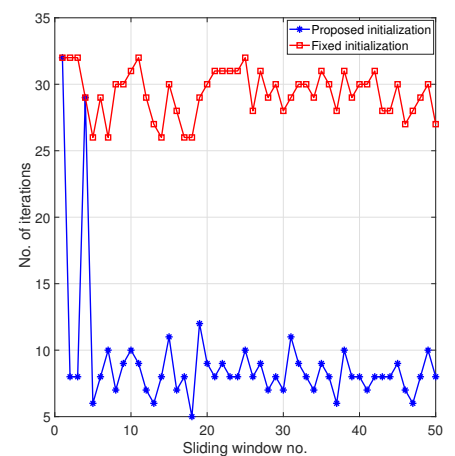

(a) No. of iterations comparison.

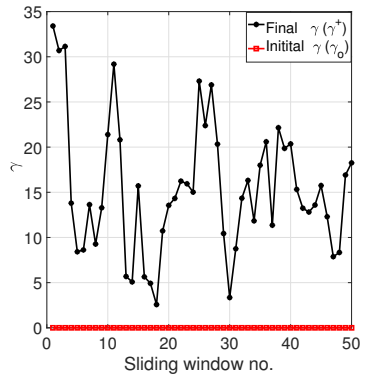

(b) Fixed initialization.

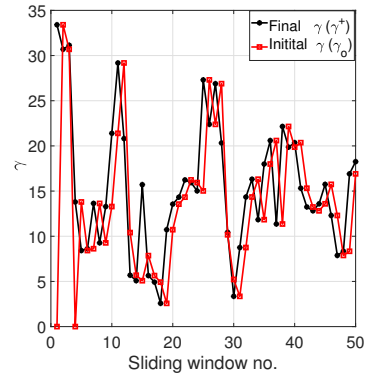

(c) Proposed initialization.
Fig. 4. Complexity comparison.

Fig. 4 (b) and (c) compare the initial values of the regularization parameter with its final values (roots) that are obtained using the fixed initialization and the proposed initialization methods, respectively. It can be seen that, contrary to fixed initialization, the initial values of the proposed technique are very close to their final values. This explains why we save a lot of iterations by using the proposed technique.

\section{CONCLUSION}

A low-complexity robust MVDR beamforming technique for a moving-source was proposed. First, a generalized sidelobe canceller (GSC) implementation was utilized to obtain an unconstrained least squares formulation of the MVDR problem. Then, the regularization parameter (diagonal loading) obtained using bounded perturbation regularization method (BPR). The regularization parameter for successive data windows was calculated in a computationally efficient way exploiting the properties of the underlying regularization method. The proposed method showed improvement in performance and reduction of simulation time by $75 \%$.

\section{REFERENCES}

[1] T. Van Le and K. Lee, "Adaptive perturbation-aided opportunistic beamforming," IEEE Communications Letters, vol. 22, no. 8, pp. 1660 1663, 2018.

[2] H. L. Van Trees, Optimum array processing: Part IV of detection, estimation, and modulation theory. John Wiley \& Sons, 2004.

[3] B. D. Van Veen and K. M. Buckley, "Beamforming: a versatile approach to spatial filtering," IEEE ASSP Magazine, vol. 5, no. 2, pp. 4-24, April 1988.
[4] Q. Nengfeng, B. Ming, H. Xiaoqing, T. Zhuanxia, and G. Luyang, "Moving target beamforming based on bayesian method," in 2015 IEEE China Summit and International Conference on Signal and Information Processing (ChinaSIP). IEEE, 2015, pp. 393-397.

[5] T. Ballal, M. A. Suliman, and T. Y. Al-Naffouri, "Bounded perturbation regularization for linear least squares estimation," IEEE Access, vol. 5, pp. 27 551-27 562, 2017.

[6] M. Suliman, T. Ballal, and T. Y. Al-Naffouri, "Robust regularized leastsquares beamforming approach to signal estimation," in 2016 IEEE Global Conference on Signal and Information Processing (GlobalSIP), Dec 2016, pp. 75-79.

[7] L. Du, J. Li, and P. Stoica, "Fully automatic computation of diagonal loading levels for robust adaptive beamforming," IEEE Transactions on Aerospace and Electronic Systems, vol. 46, no. 1, pp. 449-458, 2010.

[8] Y. Ke, C. Zheng, R. Peng, and X. Li, "Robust adaptive beamforming using noise reduction preprocessing-based fully automatic diagonal loading and steering vector estimation," IEEE Access, vol. 5, pp. 12974 $12987,2017$.

[9] Z. Tian, K. L. Bell, and H. L. Van Trees, "A recursive least squares implementation for lcmp beamforming under quadratic constraint," IEEE Transactions on Signal Processing, vol. 49, no. 6, pp. 1138-1145, 2001.

[10] S. Lu, H. Hu, X. Yu, J. Long, B. Jing, Y. Zong, and M. Wan, "Passive acoustic mapping of cavitation using eigenspace-based robust capon beamformer in ultrasound therapy," Ultrasonics sonochemistry, vol. 41, pp. 670-679, 2018.

[11] L. Huang, B. Zhang, and Z. Ye, "Robust adaptive beamforming using a new projection approach," in 2015 IEEE International Conference on Digital Signal Processing (DSP), July 2015, pp. 1181-1185.

[12] S. D. Somasundaram, N. H. Parsons, P. Li, and R. C. de Lamare, "Reduced-dimension robust capon beamforming using krylov-subspace techniques," IEEE Transactions on Aerospace and Electronic Systems, vol. 51, no. 1, pp. 270-289, January 2015.

[13] J. C. Chen, K. Yao, and R. E. Hudson, "Source localization and beamforming," IEEE Signal Processing Magazine, vol. 19, no. 2, pp. 30-39, 2002.

[14] W. Chen and X. Huang, "Wavelet-based beamforming for high-speed rotating acoustic source," IEEE Access, vol. 6, pp. 10 231-10 239, 2018.

[15] V. V. Zaharov, "Smart antenna beamforming algorithm for mobile communications with high speed moving sources," in 2008 IEEE Radio and Wireless Symposium. IEEE, 2008, pp. 279-282.

[16] I. S. Yetik and A. Nehorai, "Beamforming using the fractional fourier transform," IEEE Transactions on Signal Processing, vol. 51, no. 6, pp. 1663-1668, 2003.

[17] M. I. Ahmad, "Optimum frft domain cyclostationarity based adaptive beamforming," Signal, Image and Video Processing, pp. 1-6, 2018.

[18] S. A. Vorobyov, A. B. Gershman, and Z.-Q. Luo, "Robust adaptive beamforming using worst-case performance optimization: A solution to the signal mismatch problem," IEEE transactions on signal processing, vol. 51, no. 2, pp. 313-324, 2003.

[19] J. Li, P. Stoica, and Z. Wang, "On robust capon beamforming and diagonal loading," IEEE transactions on signal processing, vol. 51, no. 7, pp. $1702-1715,2003$.

[20] D. W. K. Ng, E. S. Lo, and R. Schober, "Robust beamforming for secure communication in systems with wireless information and power transfer,' IEEE Transactions on Wireless Communications, vol. 13, no. 8, pp. 4599-4615, 2014.

[21] V. Katkovnik, "Adaptive lpa beamforming for moving sources," Signal processing, vol. 82, no. 2, pp. 317-320, 2002.

[22] Y. Selén, R. Abrahamsson, and P. Stoica, "Automatic robust adaptive beamforming via ridge regression," Signal Processing, vol. 88, no. 1, pp. 33-49, 2008.

[23] C. J. Zarowski, An introduction to numerical analysis for electrical and computer engineers. John Wiley \& Sons, 2004.

[24] K.-B. Yu, "Recursive updating the eigenvalue decomposition of a covariance matrix," IEEE Transactions on Signal Processing, vol. 39, no. 5, pp. 1136-1145, 1991. 